\title{
Review of uptake of interventions to reduce mother to child transmission of HIV by women aware of their HIV status
}

\author{
E G Hermione Lyall, Christopher Stainsby, Graham P Taylor, Mounir Ait-Khaled, \\ Samantha Bingham, Jennifer A Evans, Anne Wright, Jonathan N Weber, Myra O McClure, \\ Sam Walters, Gareth Tudor-Williams
}

See editorial by
Mercey
Department of
Paediatrics, Imperial
College School of
Medicine at St
Mary's, London
W2 1PG
E G Hermione
Lyall,
lecturer in paediatric
infectious diseases
Jennifer A Evans,
lecturer in paediatric
infectious diseases
Sam Walters,
senior lecturer in
paediatric infectious
diseases
Gareth
Tudor-Williams,
senior lecturer in
paediatric infectious
diseases

Department of Genitourinary Medicine and Communicable Diseases, Jefferiss Research Trust Laboratories, Imperial College School of Medicine at St Mary's, London W2 1PG

Christopher Stainsby, research assistant Graham P Taylor, lecturer in

genitourinary medicine and communicable diseases

Mounir Ait-Khaled, research fellow

Samantha

Bingham, research assistant

Anne Wright, research fellow Jonathan N Weber, professor of genitourinary medicine and communicable diseases

Myra O McClure, senior lecturer in virology

Correspondence to: Dr Lyall

h.lyall@ic.ac.uk

BMJ 1998;316:268-70

\begin{abstract}
Objectives: To examine the change in uptake of interventions to reduce transmission of HIV from mothers to infants from January 1994 to July 1997. Design: Review of mother-infant pairs who presented for infant diagnosis of HIV infection.

Setting: Central London hospital with facilities for diagnosis of infant HIV infection.

Subjects: 57 consecutive mother-infant pairs, mainly from central London but also referred from surrounding hospitals.

Interventions: Data were collected on mother's country of origin; CD4 count at delivery; plasma HIV RNA copies/ml; mode of delivery; antiretroviral therapy; infant feeding; and HIV infection in infants. Main outcome measures: HIV infection of infants. Results: The vertical transmission rate was 12\% (7 pairs; 95\% confidence interval 3\% to $22 \%$ ). All mothers chose not to breast feed. The caesarean section rate was $53 \%(30 / 57)$. Antiretroviral therapy was taken by $68.5 \%(39 / 57)$ of mother-infant pairs. With antiretroviral therapy or caesarean section, or both, transmission occurred in $6 \%(0 \%$ to $13 \%)$ of pairs $(3 / 50)$. During the 24 months of 1994 and 1995, $21 \%(4 / 19)$ of infants were infected with HIV; $7.9 \%$ $(3 / 38)$ were infected over the 19 months January 1996 to July 1997 . The caesarean section rate did not change over these periods. Use of antiretroviral therapy increased from $31.5 \%(6 / 19)$ to $86.8 \%$ $(33 / 38)(\mathrm{P}<0.0001)$.

Conclusion: Women with a diagnosis of HIV infection acted to reduce the risk of transmission to their infants. Uptake of antiretroviral therapy increased significantly over time, and the caesarean section rate was persistently high.
\end{abstract}

\section{Introduction}

The Paediatric AIDS Clinical Trials Group published the results of their ACTG 076 study of zidovudine (AZT) versus placebo for prevention of vertical transmission of HIV in 1994, and use of zidovudine in pregnancy was recognised as an appropriate standard of care for prevention of vertical transmission in the United States that same year. ${ }^{12}$ This study's result has now been widely reproduced in general clinical practice and in women with more advanced HIV disease..$^{3-5}$ In the United Kingdom, use of antiretroviral therapy during pregnancy has gradually increased over the past three and a half years. However, this has been slower than in the United States or other European countries because of a low rate of antenatal diagnosis of HIV infection. ${ }^{6-7}$
We retrospectively reviewed all the mother-infant pairs presenting to our service from January 1994 to July 1997 for diagnosis of HIV infection in the infant by polymerase chain reaction and culture. The aim of this review was to examine the uptake of interventions to prevent transmission over this period. The motherinfant pairs fell into two groups: those in which mothers were known to have HIV infection in pregnancy or before and could consider options to reduce transmission; and those in which a family member, most often the infant (17 of 24), presented with HIV related symptoms. Those 24 mothers were unaware of their HIV infection antenatally and are only briefly presented here: all but one breast fed; 18 (75\%) of their infants were infected, and 11 developed AIDS during infancy.

\section{Methods}

Women known to have HIV in pregnancy were counselled about the risks of transmission and the protective effects to the infant of antiretroviral therapy, avoidance of breast feeding, and the possible protective effect of caesarean section. The mother decided whether to opt for caesarean section.

Data were collected on mother's country of origin; CD4 count at delivery; plasma HIV RNA burden before antiretroviral therapy and at delivery; mode of delivery; gestation at delivery; antiretroviral therapy regimen; infant feeding; and infection outcome for the infant. For mothers from some hospitals other than St Mary's not all data were available. Plasma concentrations of HIV RNA were measured by at least one of three commercial assays (Chiron, Organon Technica, and Roche) and the highest reading obtained was used for analysis. HIV RNA measurements were available for mothers delivering at St Mary's Hospital and for some mothers delivering elsewhere. Diagnosis of HIV infection in infants was by polymerase chain reaction of HIV DNA and HIV culture of blood mononuclear cells taken at 1 day, 4 weeks, 3 months, and 6 months of age. $^{8}$

\section{Statistical analysis}

Medians were compared by Mann-Whitney U test, and the numbers of patients in distinct groups were compared with Fisher's exact test; 95\% confidence intervals were generated treating the observed number as a Poisson variable.

\section{Results}

In 57 consecutive mother-infant pairs, $81 \%$ of mothers (46) were of African origin. Vertical transmission of HIV occurred in seven pairs $(12 \%$; $95 \%$ confidence 
interval 3\% to 22\%). Thirteen mothers were diagnosed as HIV infected during the current pregnancy, 34 were known to have HIV before this pregnancy, and the exact timing of diagnosis was unknown for 10. Eleven mothers already had a child with HIV infection. Three children developed AIDS within the first year of life. Four children were delivered prematurely, of whom one was infected.

All mothers chose not to breast feed. The caesarean rate was 53\% (30/57), including six emergencies. The rate of vertical transmission was $3 \%(1 / 30)$ by caesarean section and $22 \%(6 / 21)$ by vaginal delivery (table).

Antiretroviral therapy was taken by $69 \%(39 / 57)$ of mother-infant pairs. Twenty six $(46 \%)$ took a full 076-type course of zidovudine and seven took an incomplete course with only one or two parts of the regimen (antepartum, intrapartum, or postpartum). Six mothers took combination antiretroviral therapy antenatally (five took zidovudine plus lamivudine; one took zidovudine plus lamivudine and indinavir) and then completed the 076 course. Eighteen of the mothers $(32 \%)$ did not take or did not tolerate antiretroviral therapy. Transmission rate was 8\% (3/39) for mothers who took any kind of antiretroviral therapy and $22 \%$ $(4 / 18)$ for those who did not. In the seven pairs who took an incomplete antiretroviral course, two infants were infected. Transmission occurred in three of 50 pairs $(6 \% ; 0 \%$ to $13 \%)$ with antiretroviral therapy or caesarean section, as opposed to four of seven pairs $(57 \%)$ with vaginal delivery without antiretroviral therapy $(\mathrm{P}=0.003)$ (table).

Although this was a mainly African cohort of mothers, ethnic origin did not seem to affect mothers' choice of interventions. CD4 counts were available for only 34 mothers and HIV RNA load for 36; although this is only $60 \%$ of the group, we have no reason to suspect it is not representative of the cohort. The median CD4 count at delivery was $305 \times 10^{6} / 1$, and vertical transmission occurred at all levels. Median plasma HIV RNA was $\log 3.6$ copies $/ \mathrm{ml}$ at delivery, and transmission occurred across the range of levels. The viral load was slightly less in 26 women who took antiretroviral therapy $(\log 3.43 v \log 3.71 ; \mathrm{P}=0.06)$. Five mothers had a viral load below the level of detection of the kits $(<\log 3.0$ copies $/ \mathrm{ml})$; all took antiretroviral therapy and none transmitted HIV infection. Four of these mothers were taking combination antiretroviral therapy for either more advanced HIV disease or higher viral load at presentation.

Using the infant's date of birth as the reference point, we assessed 19 mother-infant pairs over the 24 months 1994-5 and 38 over the 19 months January 1996 to July 1997. During the first time period 21\% (4/19) of infants were infected with HIV, and $8 \%$ $(3 / 38)$ were infected in the second period. The rate of caesarean section did not change $(58 \% v 50 \%)$. Use of antiretroviral therapy increased from $32 \%(6 / 19)$ to $87 \%(33 / 38)(\mathrm{P}<0.0001)$.

\section{Discussion}

This small observational study shows that women diagnosed as HIV infected before or during pregnancy all acted to reduce the risk of transmission to their infants. No mother who knew she had HIV infection breast
Key messages

- In this London cohort, pregnant women who knew they had HIV all acted to reduce vertical transmission

- No mothers breast fed, and about half chose to have a caesarean section

- Mothers' use of antiretroviral therapy increased from $32 \%$ in $1994-5$ to $87 \%$ in $1996-7$

- With antiretroviral therapy or caesarean section, or both, vertical transmission occurred in $6 \%$ of mother-infant pairs

- Improving uptake of antenatal testing for HIV continues to be important

fed. Uptake of antiretroviral therapy increased significantly over time, and the caesarean section rate was persistently high. Mothers who received antiretroviral therapy or caesarean section, or both, had a transmission rate of $6 \%$; the overall transmission rate for the group was $12 \%$. This cohort is too small to study the separate effects of variables such as viral load, CD4 count, antiretroviral therapy, and caesarean section on transmission. HIV transmission occurred at all levels of viral load and CD4 count; this concurs with the findings of the 076 study, where zidovudine did not alter this pattern but reduced transmission at all levels. ${ }^{9}$

Our findings complement those of an earlier study of uptake of interventions in the United Kingdom that showed a continued increase in uptake of interventions by mothers. ${ }^{7}$ In our study ethnic group did not affect mothers' choice of interventions.

With improved antenatal detection of HIV infection and use of antiretroviral therapy, vertical transmission should greatly decline. This will result in increased exposure of uninfected infants to potent drugs in utero and around the time of birth. Although there is no evidence of short term deleterious effects of zidovudine, the children from the 076 study will be followed to adulthood to monitor late effects. There is much less data on effects of other drugs now being used in pregnancy. Clinicians prescribing antiretroviral therapy should report to the International Registry of Antiretroviral Use in Pregnancy (c/o Glaxo Wellcome, Greenford, Middlesex UB6 OHE) so that information can be gathered on the increasing number of exposed but uninfected children.

We are grateful to St Mary's Hospital Trust midwives, especially Ms S Dick, Ms M Hornby, and Ms F Clayton, for their assistance with the study and to specialist nurse Paula Seery, Chelsea and Westminster Hospital, for collection of data. We are grateful to

Use of preventive interventions and vertical transmission of HIV in 57 mothers known to be infected before delivery

\begin{tabular}{|c|c|c|c|}
\hline Intervention & $\begin{array}{c}\text { Infant infected with } \\
\text { HIV }\end{array}$ & $\begin{array}{l}\text { Infant not } \\
\text { infected }\end{array}$ & $P$ value ${ }^{*}$ \\
\hline Antiretroviral therapy & 3 & 36 & \multirow{2}{*}{0.26} \\
\hline No antiretroviral therapy & 4 & 14 & \\
\hline Caesarean section $\dagger$ & 1 & 29 & \multirow{2}{*}{0.074} \\
\hline Vaginal delivery & 6 & 21 & \\
\hline Antiretroviral therapy or caesarean section, or both & 3 & 47 & \multirow{2}{*}{0.003} \\
\hline No antiretroviral therapy and vaginal delivery & 4 & 3 & \\
\hline
\end{tabular}

*Fisher's exact test.

†Elective and emergency caesarean sections. 
clinicians in the following hospitals for allowing us to study their patients: Chelsea and Westminster, Hillingdon, Central Middlesex, North Middlesex, West Middlesex, Milton Keynes, Northampton General, Northwick Park, Royal Free, and Watford General. Thanks to R Booy for fruitful discussions.

Contributors: JNW, MOMcC, JAE, and SW set up and have monitored the infant HIV diagnostic service. JNW and GT-W were the instigators of this paper. EGHL wrote the paper and CS, GPT, JNW, MOMcC, SW, and GT-W took part in its editing. $\mathrm{CS}, \mathrm{MA}-\mathrm{K}$, and SB carried out laboratory testing of the infants and mothers. EGHL, GPT, and CS collated the results; Paula Seery collated results from the Chelsea and Westminster Hospital. Contributors from the clinical paediatric service were SW, GT-W, JAE, Stephen Marriage, and EGHL; contributors from the clinical adult service were GPT, Siobhan Crowley, and AW; contributors from obstetrics and midwifery were Ms S Dick, Ms M Hornby, Ms F Clayton, and Mr J Smith. Other clinicians from surrounding hospitals who referred their patients also contributed clinically (see acknowledgements). The guarantor is Hermione Lyall.

Funding: No additional funding.

Conflict of interest: None.

1 Connor EM, Sperling RS, Gelber R, Kiselev P, Scott G, O'Sullivan MJ, et al for the Paediatric AIDS Clinical Trials Group Protocol 076 Study
Group. Reduction of maternal-infant transmission of HIV-1 with zidovudine treatment. N Engl J Med 1994;331:1173-80.

2 Centres for Disease Control. Public Health Service task force on use of zidovudine to reduce perinatal transmission of human immunodeficiency virus. MMWR 1994;43(RR-11):1-21.

3 Fiscus SA, Adimora AA, Schoenbach VJ, Lim W, Mckinney R, Rupar D, et al. Perinatal HIV infection and the effect of zidovudine therapy on transmission in rural and urban counties. JAMA 1996:275;1483-8.

4 Blanche S, Mayaux MJ, Mandelbrot L, Rouzioux C, Delfraissy JF for the French Pediatric HIV Infection Study Group. Acceptability and impact of zidovudine prevention on mother to child HIV-1 transmission in France [abstract]. 4th International Conference on Retroviruses and Opportunistic Infections, Washington, DC, 1997.

5 National Institute of Child Health and Human Development. Pediatric ACTG protocol 185. Executive summary. Bethesda, MD: National Institutes of Health, 1997.

6 Department of Health. Unlinked anomymous HIV prevalence monitoring programme: England and Wales. Data to end 1995. London: DoH, 1996.

7 Gibb DM, MacDonagh SE, Tookey PA, Duong T, Nicoll A, Goldberg DJ, et al. Uptake of interventions to reduce mother-to-infant transmission of HIV in the United Kingdom and Ireland. AIDS 1997;11:F53-8.

8 McClure MO, Bieniasz PD, Weber JN, Tedder RS, O'Shea S, Banatvala JE, et al. HIV clearance in an infant? Nature 1995;375:637.

9 Sperling RS, Shapiro DE, Coombs RW, Todd JA, Herman SA, McSherry GD, et al. Maternal viral load, zidovudine treatment, and the risk of transmission of HIV-1 from mother to infant. N Engl J Med 1996;335:1621-9

\title{
Antenatal HIV testing: current problems, future solutions. Survey of uptake in one London hospital
}

\author{
Teresa A Duffy, Charles D A Wolfe, Claire Varden, Jane Kennedy, Ian L Chrystie, Jangu E Banatvala
}

See editorial by

Mercey

Department of

Midwifery, Guy's

and St Thomas's

NHS Trust, St

Thomas's Hospital,

London SE1 7EH

Teresa A Duffy,

research midwife

Jane Kennedy,

midwife counsellor

Public Health

Medicine, United

Medical and Dental

Schools of Guy's

and St Thomas's

Hospitals, London

SE1 7EH

Charles D A Wolfe,

senior lecturer

Claire Varden,

assistant statistician

Department of

Virology, United

Medical and Dental

Schools of Guy's

and St Thomas's

Hospitals, London

SE1 7EH

Ian L Chrystie,

lecturer

Jangu E Banatvala,

professor of clinical

virology

Correspondence to: Professor Banatvala

BMJ 1998;316:270-1
Pregnant women attending Guy's and St Thomas's Hospitals Trust have one of the highest prevalence rates for HIV-1 in inner London (0.53\% in 1996). ${ }^{1}$ In 1992 we showed that this was associated with African ethnic origin. ${ }^{2}$ However, despite the Department of Health's recommendations that named HIV testing be made available to all pregnant women in areas of relatively high prevalence, uptake in our trust is disappointingly low-about 30\%-as elsewhere in inner London. In 1995, throughout London, only 26 of 205 (13\%) HIV positive pregnant women had been identified antenatally. ${ }^{3}$ Most were therefore almost certainly unable to benefit from recent advances in treatment and in the prevention of mother to child transmission of HIV.

\section{Subjects, methods, and results}

This paper describes uptake of HIV testing among pregnant women between 1991 and 1996 and includes a detailed survey of 789 women, of whom 428 attended antenatal clinics at Guy's Hospital, 310 attended six community clinics, and 51 attended a midwifery group practice between 5 March and 20 December 1996. Of the antenatal population, $50 \%$ were white, $24 \%$ were black African, and 13\% were black Caribbean. Before booking, women were sent a locally produced leaflet about HIV. At booking, midwives-49 of $51(96 \%)$ of whom had had specific training about HIV-initiated a pretest discussion, and before and after booking women were invited to complete questionnaires relating to attitudes towards HIV testing.

Although 35\% of 789 women accepted the offer of an HIV test, over a third of those who had intended to be tested changed their mind during the booking interview; only a few of those not intending to be tested did so (table). Multivariate analysis showed that being non-white was a significant predictor of uptake $(\mathrm{P}=0.044)$ and that uptake was higher in the hospital based clinic (41\%) than in the community clinic (30\%) or midwifery group practice $(10 \%)(\mathrm{P}=0.0001)$.

\section{Comment}

Since the start of antenatal HIV testing in 1985, uptake at St Thomas's Hospital has risen from 5\% to about $30 \%$. In 1992-4, of those identified on a named basis, $14 / 18(78 \%)$ were identified via the genitourinary medicine clinic; in 1995-6, 19 of 32 (65\%) were identified in antenatal clinics.

Although our results may seem disappointing in terms of uptake, the value of midwifery staff trained in HIV is emphasised by differences in HIV detection rates between Guy's and St Thomas's Hospitals before their unification in 1995. Between 1991 and 1995, Guy's, which had no midwifery staff trained in HIV, failed to identify any of the HIV positive pregnant women identified by the Public Health Laboratory Service's unlinked anonymous survey; at St Thomas's, however, which had an HIV specialist midwife counsellor and trained staff, 39 of $105(37 \%)$ were identified.

Merely achieving higher uptake rates may not increase the proportion of women positive for HIV-1 identified, as many may decline testing. Indeed, in the past 8 months, we know of three pregnant women who knew they were HIV positive at booking but declined testing without divulging their serostatus. 